\title{
THE QUANTUM HYDRODYNAMIC MODEL FOR SEMICONDUCTOR DEVICES*
}

\author{
CARL L. GARDNER ${ }^{\dagger}$
}

\begin{abstract}
The classical hydrodynamic equations can be extended to include quantum effects by incorporating the first quantum corrections. These quantum corrections are $O\left(\hbar^{2}\right)$.

The full three-dimensional quantum hydrodynamic (QHD) model is derived for the first time by a moment expansion of the Wigner-Boltzmann equation. The QHD conservation laws have the same form as the classical hydrodynamic equations, but the energy density and stress tensor have additional quantum terms. These quantum terms allow particles to tunnel through potential barriers and to build up in potential wells.

The three-dimensional QHD transport equations are mathematically classified as having two Schrödinger modes, two hyperbolic modes, and one parabolic mode. The one-dimensional steady-state QHD equations are discretized in conservation form using the second upwind method.

Simulations of a resonant tunneling diode are presented that show charge buildup in the quantum well and negative differential resistance (NDR) in the current-voltage curve. These are the first simulations of the full QHD equations to show NDR in the resonant tunneling diode. The computed current-voltage curve agrees quantitatively with experimental measurements. NDR is interpreted in terms of the time spent by electrons in the quantum well.
\end{abstract} simulation

Key words. quantum hydrodynamics, nonlinear PDEs, conservation laws, semiconductor device

AMS subject classifications. 76M20, 76W05, 76Y05

1. Introduction. The behavior of a quantum fluid near thermal equilibrium and in the high temperature limit can be approximated by adding $O\left(\hbar^{2}\right)$ corrections to the classical fluid dynamical equations. The $O\left(\hbar^{2}\right)$ terms allow particle tunneling through potential barriers and particle buildup in potential wells.

The primary application of various quantum hydrodynamic (QHD) equations to date has been in analyzing the flow of electrons in quantum semiconductor devices. The QHD equations, however, are quite general and may find applications in other areas of physics, e.g., fluid models of the nucleus, superconductivity, and superfluidity.

In this investigation, we derive the QHD equations from a moment expansion of the Wigner-Boltzmann equation [31] and solve the QHD equations numerically to simulate electron flow in the resonant tunneling diode.

The QHD model is introduced in $\$ 2$, and the first derivation of the full three-dimensional (3D) QHD equations from a moment expansion of the Wigner-Boltzmann equation is presented in $\$ 3$. The QHD transport equations are expressed in terms of conservation laws for particles, momentum, and energy. The quantum correction to the stress tensor appearing in the QHD equations was derived by Ancona, Iafrate, and Tiersten [1], [2]. In the one-dimensional (1D) case, the 3D QHD equations reduce to the QHD model of Grubin and Kreskovsky [14].

* Received by the editors November 23, 1992; accepted for publication (in revised form) June 18, 1993. The research of the author was supported in part by U.S. Army Research Office grant DAAL03-91-G-0146.

${ }^{\dagger}$ Department of Computer Science and Department of Mathematics, Duke University, Durham, North Carolina 27706. 
A different set of 3D QHD equations was proposed in [34]. However, this QHD model does not agree with the QHD equations derived here from the $O\left(\hbar^{2}\right)$ thermal equilibrium Wigner distribution function, that, we believe, must give the leading behavior of the quantum corrections.

In $\S 4$ the 3D QHD equations are classified as having two Schrödinger modes, two hyperbolic modes, one parabolic mode, and one elliptic mode, and well-posed boundary conditions are specified for the $1 \mathrm{D}$ resonant tunneling diode. The second upwind discretization of the 1D QHD equations is presented in $\$ 5$.

Numerical simulations of a resonant tunneling diode were presented in [14] and [32] using the 1D QHD model. The simulations in [14] show negative differential resistance (NDR) for a resonant tunneling diode at $77 \mathrm{~K}$ using only the first two moments (1) and (2) plus Poisson's equation. (Reference [32] applies the full 1D QHD model in simulating a resonant tunneling diode at $300 \mathrm{~K}$, but fails to find NDR.) In $\$ 6$ the first simulations of the full 1D QHD equations are presented that show NDR in the resonant tunneling diode. An analysis of electron behavior in the diode is given in terms of the fluid dynamical quantities density, velocity, and temperature. The computed current-voltage curve for the resonant tunneling diode agrees quantitatively with experimental measurements. Finally, NDR is interpreted in terms of the time spent by electrons in the quantum well (see [14]).

2. The QHD model. The QHD conservation laws have the same form as the classical hydrodynamic equations (for simplicity, we treat the flow of electrons in an electric field)

$$
\begin{aligned}
\frac{\partial n}{\partial t}+\frac{1}{m} \frac{\partial \Pi_{i}}{\partial x_{i}} & =0 \\
\frac{\partial \Pi_{j}}{\partial t}+\frac{\partial}{\partial x_{i}}\left(u_{i} \Pi_{j}-P_{i j}\right) & =-n \frac{\partial V}{\partial x_{j}}-\frac{\Pi_{j}}{\tau_{p}} \\
\frac{\partial W}{\partial t}+\frac{\partial}{\partial x_{i}}\left(u_{i} W-u_{j} P_{i j}+q_{i}\right) & =-\frac{\Pi_{i}}{m} \frac{\partial V}{\partial x_{i}}-\frac{\left(W-\frac{3}{2} n T_{0}\right)}{\tau_{w}}
\end{aligned}
$$

where $n$ is the electron density, $\mathbf{u}$ is the velocity, $\Pi_{i}$ is the momentum density, $P_{i j}$ is the stress tensor, $V=-e \phi$ is the potential energy, $\phi$ is the electric potential, $e>0$ is the electronic charge, $W$ is the energy density, $\mathbf{q}$ is the heat flux, and $T_{0}$ is the temperature of the semiconductor lattice in energy units $\left(k_{B}\right.$ is set equal to 1$)$. Indices $i, j, k, l$ equal $1,2,3$, and repeated indices are summed over using the Einstein convention. Equation (1) expresses conservation of electron number, (2) expresses conservation of momentum, and (3) expresses conservation of energy. The last terms in (2) and (3) represent electron scattering, ${ }^{1}$ which is modeled by the standard relaxation time approximation with momentum and energy relaxation times $\tau_{p}$ and $\tau_{w}$.

The classical hydrodynamic equations can be derived from physical arguments or from a moment expansion of the Boltzmann equation. ${ }^{2}$ The quantum conservation laws have the same form as their classical counterparts. However, to close the

\footnotetext{
${ }^{1}$ The collision terms may include the effects of electron-phonon and electron-impurity collisions, intervalley and interband scattering, etc.

${ }^{2}$ Whitham [30] gives a clear account of the physical arguments leading to the classical hydrodynamic equations. For a derivation of the hydrodynamic model for semiconductor devices from the Boltzmann equation, see [25].
} 
moment expansion at three moments, we must define, for example, $\Pi_{i}, P_{i j}, W$, and $\mathbf{q}$ in terms of $n, \mathbf{u}$, and $T$, where $T$ is the electron temperature.

We assume that the heat flux is specified by the Fourier law $\mathbf{q}=-\kappa \nabla T$. Section 3 shows that, for the $O\left(\hbar^{2}\right)$ "momentum-shifted" thermal equilibrium Wigner distribution function, the momentum density $\Pi_{i}=m n u_{i}$, where $m$ is the effective electron mass, the stress tensor ${ }^{3}$ is given by

$$
P_{i j}=-n T \delta_{i j}+\frac{\hbar^{2} n}{12 m} \frac{\partial^{2}}{\partial x_{i} \partial x_{j}} \log (n)+O\left(\hbar^{4}\right),
$$

and the energy density is given by

$$
W=\frac{3}{2} n T+\frac{1}{2} m n u^{2}-\frac{\hbar^{2} n}{24 m} \nabla^{2} \log (n)+O\left(\hbar^{4}\right) .
$$

The quantum correction to the energy density was first derived by Wigner [31]. The quantum correction to the stress tensor was proposed by Ancona and Tiersten [2] on general thermodynamical grounds and derived by Ancona and Iafrate [1] in the Wigner formalism. The stress tensor is related to the quantum potential of Bohm [22]

$$
Q=-\frac{\hbar^{2}}{2 m} \frac{1}{\sqrt{n}} \nabla^{2} \sqrt{n}
$$

through the formula

$$
-\frac{\partial}{\partial x_{i}} P_{i j}=\frac{\partial}{\partial x_{j}}(n T)+\frac{n}{3} \frac{\partial Q}{\partial x_{j}} .
$$

The transport equations (1)-(3) are coupled to Poisson's equation for the electric potential energy

$$
\nabla \cdot(\epsilon \nabla V)=e^{2}\left(N_{D}-N_{A}-n\right),
$$

where $\epsilon$ is the dielectric constant, $N_{D}$ is the density of donors, and $N_{A}$ is the density of acceptors.

3. Moment expansion of the Wigner-Boltzmann equation. In this section, the QHD equations are derived as a set of nonlinear conservation laws by a moment expansion of the Wigner-Boltzmann equation [31] and an expansion of the thermal equilibrium Wigner distribution function to $O\left(\hbar^{2}\right)$.

To derive the QHD equations, we assume that the electron flow can be approximated by a single-particle effective mass Schrödinger equation with a self-consistent many-body field,

$$
i \hbar \frac{\partial \psi_{\lambda}}{\partial t}=E_{\lambda} \psi_{\lambda}=-\frac{\hbar^{2}}{2 m} \nabla^{2} \psi_{\lambda}+V \psi_{\lambda}
$$

where the wavefunctions $\psi_{\lambda}(\mathbf{x}, t)(\lambda=1,2, \ldots)$ have energies $E_{\lambda}$ and where $V(\mathbf{x})$ is the self-consistent potential energy. The effective mass approximation states that Schrödinger's equation with the free electron mass and the total potential energy, which includes the periodic potential energy of the semiconductor lattice plus $V$, can

\footnotetext{
${ }^{3}$ The stress tensor is symmetric due to conservation of angular momentum.
} 
be rewritten with the total potential energy replaced by $V$ and the electron mass replaced by the effective electron mass (see, e.g., [19, Chap. 2]). Many-body electron correlation effects are neglected in this theory. Also neglected are the effects of Fermi-Dirac statistics, although these effects can be included. These approximations are valid in semiconductors in the low density $\left(\leq 10^{19} \mathrm{~cm}^{-3}\right)$ and high temperature ( $\gtrsim 77 \mathrm{~K}$ ) limit. We also assume that the fluid dynamical state variables $n$, $\mathbf{u}$, and $T$ are slowly varying functions of $\mathbf{x}$ and $t$.

For a pure quantum mechanical state described by a wavefunction $\psi(\mathbf{x}, t)$, the Wigner distribution function is defined as

$$
f_{W}(\mathbf{x}, \mathbf{p})=(\pi \hbar)^{-3} \int d^{3} y \psi^{*}(\mathbf{x}+\mathbf{y}) \psi(\mathbf{x}-\mathbf{y}) e^{2 i \mathbf{p} \cdot \mathbf{y} / \hbar}
$$

where $\mathbf{p}$ is the molecular momentum. (The time dependence in $\psi, f_{W}$, etc., will be left implicit except when needed for clarity. All integrals are from $-\infty$ to $+\infty$.)

For a mixed quantum mechanical state described by wavefunctions $\psi_{\lambda}(\mathbf{x}, t)$ with occupation numbers $a_{\lambda}$, the Wigner distribution function is defined as

$$
f_{W}(\mathbf{x}, \mathbf{p})=(\pi \hbar)^{-3} \sum_{\lambda} a_{\lambda} \int d^{3} y \psi_{\lambda}^{*}(\mathbf{x}+\mathbf{y}) \psi_{\lambda}(\mathbf{x}-\mathbf{y}) e^{2 i \mathbf{p} \cdot \mathbf{y} / \hbar}
$$

Here $\sum_{\lambda} a_{\lambda}=M$, where $M$ is the total number of electrons in the system. We assume that the $a_{\lambda}$ 's vary slowly with $\mathbf{x}$ and $t$. For a mixed state in thermal equilibrium with a heat bath at temperature $T \equiv 1 / \beta, a_{\lambda} \propto e^{-\beta E_{\lambda}}$. Equations (10) and (11) are the Fourier transforms of the quantum mechanical density matrices for pure and mixed states, respectively. "Global" expectation values of observables $A$ are given in the Wigner formalism by

$$
\langle A\rangle_{\text {global }}=\int d^{3} x d^{3} p A(\mathbf{x}, \mathbf{p}) f_{W}(\mathbf{x}, \mathbf{p})
$$

When $A(\mathbf{x}, \mathbf{p})$ is the sum of a function of $\mathbf{p}$ and a function of $\mathbf{x}$ (all physically important operators including the Hamiltonian are of this form), (12) is equivalent to

$$
\langle A\rangle_{\text {global }}=\int d^{3} x \psi^{*} A\left(\mathbf{x}, \frac{\hbar}{i} \nabla\right) \psi
$$

for a pure state and

$$
\langle A\rangle_{\text {global }}=\sum_{\lambda} a_{\lambda} \int d^{3} x \psi_{\lambda}^{*} A\left(\mathbf{x}, \frac{\hbar}{i} \nabla\right) \psi_{\lambda}
$$

for a mixed state, where the wavefunctions are normalized so that $\int d^{3} x \psi^{*} \psi=1$ and $\int d^{3} x \psi_{\lambda}^{*} \psi_{\lambda}=1$.

Thus $f_{W}$ plays a role in quantum mechanics analogous to that of the Boltzmann distribution function in classical mechanics. However, the Wigner distribution function cannot be interpreted as a classical probability since it can take on negative values. "Local" average values of $A$ are here defined in the Wigner formalism as

$$
\langle A(x)\rangle=\int d^{3} p A(\mathbf{x}, \mathbf{p}) f_{W}(\mathbf{x}, \mathbf{p})
$$


The Wigner distribution function obeys an equation similar to the classical Boltzmann equation

$$
\begin{aligned}
\frac{\partial f_{W}}{\partial t} & +\frac{\mathbf{p}}{m} \cdot \nabla f_{W} \\
& -\sum_{\lambda_{1}, \lambda_{2}, \lambda_{3}=0}^{\infty} \frac{(\hbar / 2 i)^{\lambda_{1}+\lambda_{2}+\lambda_{3}-1}}{\lambda_{1} ! \lambda_{2} ! \lambda_{3} !} \frac{\partial^{\lambda_{1}+\lambda_{2}+\lambda_{3}} V}{\partial x_{1}^{\lambda_{1}} \partial x_{2}^{\lambda_{2}} \partial x_{3}^{\lambda_{3}}} \frac{\partial^{\lambda_{1}+\lambda_{2}+\lambda_{3}} f_{W}}{\partial p_{1}^{\lambda_{l}} \partial p_{2}^{\lambda_{2}} \partial p_{3}^{\lambda_{3}}}=0
\end{aligned}
$$

with $\lambda_{1}+\lambda_{2}+\lambda_{3}$ an odd integer, or in compact form

$$
\frac{\partial f_{W}}{\partial t}+\frac{\mathbf{p}}{m} \cdot \nabla f_{W}-\frac{2}{\hbar} \sin \left(\frac{\hbar}{2} \mathbf{V}_{\mathbf{x}}^{V} \cdot \nabla_{\mathbf{p}}\right) V(\mathbf{x}) f_{W}(\mathbf{x}, \mathbf{p})=0
$$

where $\nabla_{\mathbf{x}}^{V}$ acts only on $V$. (We assume that the potential energy $V$ has a Taylor series expansion. If $V$ does not have a Taylor series expansion, the integrodifferential form [31] of the Wigner-Boltzmann equation can be used.) Equation (16) is known as the Wigner-Boltzmann equation. In the limit $\hbar \rightarrow 0$, (16) reduces to the classical collisionless Boltzmann equation. The Wigner-Boltzmann equation follows directly from Schrödinger's equation and represents an exact reformulation of quantum mechanics (in its $6 M$-dimensional form, with the full potential energy and the free electron mass).

To derive (16) from Schrödinger's equation (9), first differentiate (11) with respect to $t$ as follows:

$$
\begin{aligned}
i \hbar \frac{\partial f_{W}}{\partial t}=\sum_{\lambda} a_{\lambda} \int \frac{d^{3} y}{(\pi \hbar)^{3}} e^{2 i \mathbf{p} \cdot \mathbf{y} / \hbar}\left\{\frac{\hbar^{2}}{2 m}[\right. & \left\{\nabla_{\mathbf{x}}^{2} \psi_{\lambda}^{*}(\mathbf{x}+\mathbf{y})\right\} \psi_{\lambda}(\mathbf{x}-\mathbf{y}) \\
& \left.-\psi_{\lambda}^{*}(\mathbf{x}+\mathbf{y}) \nabla_{\mathbf{x}}^{2} \psi_{\lambda}(\mathbf{x}-\mathbf{y})\right] \\
& \left.-[V(\mathbf{x}+\mathbf{y})-V(\mathbf{x}-\mathbf{y})] \psi_{\lambda}^{*}(\mathbf{x}+\mathbf{y}) \psi_{\lambda}(\mathbf{x}-\mathbf{y})\right\},
\end{aligned}
$$

where $a_{\lambda}$ is considered constant for the derivation. Then, by replacing $\nabla_{\mathbf{x}}$ with $\nabla_{\mathbf{y}}$ and integrating the first two terms on the right-hand side of (18) by parts, we obtain

$$
\begin{aligned}
\frac{\partial f_{W}}{\partial t}=\sum_{\lambda} & a_{\lambda} \int \frac{d^{3} y}{(\pi \hbar)^{3}} e^{2 i \mathbf{p} \cdot \mathbf{y} / \hbar}\left\{\frac { \mathbf { p } } { m } \cdot \left[-\left\{\nabla_{\mathbf{y}} \psi_{\lambda}^{*}(\mathbf{x}+\mathbf{y})\right\} \psi_{\lambda}(\mathbf{x}-\mathbf{y})\right.\right. \\
& \left.+\psi_{\lambda}^{*}(\mathbf{x}+\mathbf{y}) \nabla_{\mathbf{y}} \psi_{\lambda}(\mathbf{x}-\mathbf{y})\right] \\
& \left.+\frac{2 i}{\hbar} \sum_{\lambda_{1}, \lambda_{2}, \lambda_{3}} \frac{y_{1}^{\lambda_{1}} y_{2}^{\lambda_{2}} y_{3}^{\lambda_{3}}}{\lambda_{1} ! \lambda_{2} ! \lambda_{3} !} \frac{\partial^{\lambda_{1}+\lambda_{2}+\lambda_{3}} V}{\partial x_{1}^{\lambda_{1}} \partial x_{2}^{\lambda_{2}} \partial x_{3}^{\lambda_{3}}} \psi_{\lambda}^{*}(\mathbf{x}+\mathbf{y}) \psi_{\lambda}(\mathbf{x}-\mathbf{y})\right\}
\end{aligned}
$$

with $\lambda_{1}+\lambda_{2}+\lambda_{3}$ an odd integer and where $V(\mathbf{x} \pm \mathbf{y})$ has been expanded in a Taylor series. Replacing $\nabla_{\mathbf{y}}$ with $\nabla_{\mathbf{x}}$ leads to the Wigner-Boltzmann equation (16).

The moment expansion of the Wigner-Boltzmann equation involves integrating powers of $p_{j}$ against $f_{W}(\mathbf{x}, \mathbf{p})$ in (16) to obtain conservation laws for particle number, momentum, and energy [15], [27]. Integrating a function $\chi(\mathbf{p})$ with respect to $\mathbf{p}$ against 
the Wigner-Boltzmann equation, we obtain

$$
\begin{aligned}
& \frac{\partial\langle\chi\rangle}{\partial t}+\nabla \cdot\left\langle\frac{\mathbf{p}}{m} \chi\right\rangle \\
& \quad+\sum_{\lambda_{1}, \lambda_{2}, \lambda_{3}} \frac{(\hbar / 2 i)^{\lambda_{1}+\lambda_{2}+\lambda_{3}-1}}{\lambda_{1} ! \lambda_{2} ! \lambda_{3} !} \frac{\partial^{\lambda_{1}+\lambda_{2}+\lambda_{3}} V}{\partial x_{1}^{\lambda_{1}} \partial x_{2}^{\lambda_{2}} \partial x_{3}^{\lambda_{3}}}\left\langle\frac{\partial^{\lambda_{1}+\lambda_{2}+\lambda_{3}} \chi}{\partial p_{1}^{\lambda_{1}} \partial p_{2}^{\lambda_{2}} \partial p_{3}^{\lambda_{3}}}\right\rangle=0
\end{aligned}
$$

with $\lambda_{1}+\lambda_{2}+\lambda_{3}$ an odd integer.

Setting $\chi$ equal to $1, p_{j}$, and $p^{2} / 2 m$ yields the first three moment equations

$$
\begin{aligned}
\frac{\partial n}{\partial t}+\frac{1}{m} \frac{\partial\left\langle p_{i}\right\rangle}{\partial x_{i}} & =0 \\
\frac{\partial\left\langle p_{j}\right\rangle}{\partial t}+\frac{\partial}{\partial x_{i}}\left\langle\frac{p_{i} p_{j}}{m}\right\rangle & =-n \frac{\partial V}{\partial x_{j}}, \\
\frac{\partial}{\partial t}\left\langle\frac{p^{2}}{2 m}\right\rangle+\frac{\partial}{\partial x_{i}}\left\langle\frac{p_{i} p^{2}}{2 m^{2}}\right\rangle & =-\frac{1}{m}\left\langle p_{i}\right\rangle \frac{\partial V}{\partial x_{i}} .
\end{aligned}
$$

The quantum conservation laws have the same form as their classical counterparts. Explicit factors of $\hbar$ enter only at the fourth $\left(\chi=\left\langle p_{j} p^{2} / 2 m^{2}\right\rangle\right)$ and higher moments due to the structure of the average value in the third term on the left-hand side of (20).

To calculate the average values in the first three moment equations, we use the "momentum-shifted" version of the $O\left(\hbar^{2}\right)$ solution [31] to (16)

$$
\begin{aligned}
f_{W}^{(2)}(\mathbf{x}, \mathbf{p})= & \mathscr{A} \frac{e^{-\beta E}}{(2 \pi \hbar)^{3}} \\
& \times\left[1+\hbar^{2}\left\{-\frac{\beta^{2}}{8 m} \frac{\partial^{2} V}{\partial x_{k}^{2}}+\frac{\beta^{3}}{24 m}\left(\frac{\partial V}{\partial x_{k}}\right)^{2}+\frac{\beta^{3} p_{k}^{\prime} p_{l}^{\prime}}{24 m^{2}} \frac{\partial^{2} V}{\partial x_{k} \partial x_{l}}\right\}+O\left(\hbar^{4}\right)\right],
\end{aligned}
$$

where $\mathscr{A}$ is a slowly varying function of $\mathbf{x}$ and $t$, the classical nonrelativistic energy $E=p^{\prime 2} / 2 m+V$, and the macroscopic fluid velocity $\mathbf{u}$ is related to the molecular momentum $\mathbf{p}$ by

$$
\mathbf{p}=m \mathbf{u}+\mathbf{p}^{\prime}
$$

The actual expansion parameter in the asymptotic series (24) is $\hbar^{2} / 8 m T l^{2}$, where $l$ is a characteristic length scale of the problem [1], [26].

The leading term in $f_{W}^{(2)}$ as $\hbar \rightarrow 0$ is the classical Maxwell-Boltzmann distribution function. With $\mathbf{u}=0, f_{W}^{(2)}$ is the thermal equilibrium solution to the Wigner-Boltzmann equation to $O\left(\hbar^{2}\right)$. We then assume that the momentum-shifted $f_{W}^{(2)}$ approximates the actual Wigner distribution function closely enough for the average values in (21)-(23) to be close to the actual values.

Equation (24) for the Wigner distribution function has five free parameters $\mathscr{A}$ (which is proportional to $n$ ), $\mathbf{u}$, and $1 / \beta=T$, which correspond to the five unknowns in the hydrodynamic transport equations (1)-(3). 
The integral of $f_{W}$ with respect to $\mathbf{p}$ is the particle density $n(\mathbf{x}, t)$ (which equals $\psi^{*} \psi$ for a pure state and $\Sigma_{\lambda} a_{\lambda} \psi_{\lambda}^{*} \psi_{\lambda}$ for a mixed state)

$$
\begin{aligned}
n(\mathbf{x}, t) & =\int d^{3} p f_{W}(\mathbf{x}, \mathbf{p}) \approx \int d^{3} p^{\prime} f_{W}^{(2)}(\mathbf{x}, \mathbf{p}) \\
& =C e^{-\beta V}\left[1+\hbar^{2}\left\{-\frac{\beta^{2}}{12 m} \frac{\partial^{2} V}{\partial x_{k}^{2}}+\frac{\beta^{3}}{24 m}\left(\frac{\partial V}{\partial x_{k}}\right)^{2}\right\}+O\left(\hbar^{4}\right)\right],
\end{aligned}
$$

where $C=\left(m / 2 \pi \beta \hbar^{2}\right)^{3 / 2} \mathscr{A}$.

Using the shifted Wigner distribution function (24) and expanding $\mathbf{p}$ according to (25), we obtain

$$
\begin{gathered}
\left\langle p_{i}\right\rangle \equiv \Pi_{i}=m n u_{i}, \\
\left\langle\frac{p_{i} p_{j}}{m}\right\rangle=u_{i} \Pi_{j}-P_{i j}, \quad P_{i j} \equiv-\left\langle\frac{p_{i}^{\prime} p_{j}^{\prime}}{m}\right\rangle, \\
\left\langle\frac{p^{2}}{2 m}\right\rangle \equiv W, \quad W=\frac{1}{2} m n u^{2}+\left\langle\frac{p^{\prime 2}}{2 m}\right\rangle, \\
\left\langle\frac{p_{i} p^{2}}{2 m^{2}}\right\rangle=u_{i} W-u_{j} P_{i j}+q_{i}, \quad q_{i} \equiv\left\langle\frac{p_{i}^{\prime} p^{\prime 2}}{2 m^{2}}\right\rangle .
\end{gathered}
$$

We used the fact that odd powers of $p_{i}^{\prime}$ against $f_{W}^{(2)}$ integrate to zero, except that a heat flux term $\mathbf{q}$ is allowed, since heat conduction plays an important role in both the classical and QHD models. (The heat conduction term, in its Fourier form $\mathbf{q}=-\kappa \nabla T$, is believed to incorporate the most important effects of non-Maxwellian distributions. Extended models for $\mathbf{q}$ can also represent important effects of higher moments; see [33].) Note that $\Pi_{i}=m n u_{i}$ is exact to all orders in $\hbar$ for the Wigner distribution function given by (24). The definitions in (27)-(30) in terms of the Wigner distribution function have the same form as the classical definitions (see [30]) in terms of the Boltzmann distribution function. Substituting the expressions (27)-(30) into the moment equations (21)-(23), we derive the collisionless QHD model ((1)-(3) with $\tau_{p}$, $\left.\tau_{w} \rightarrow \infty\right)$.

Finally, to calculate $P_{i j}$ and $W$, first observe that

$$
\begin{gathered}
\int d^{3} p^{\prime} \frac{p_{i}^{\prime} p_{j}^{\prime}}{m} f_{W}^{(2)}(\mathbf{x}, \mathbf{p}) \\
=\frac{C}{\beta} e^{-\beta V}\left[\delta_{i j}+\hbar^{2}\left\{\delta_{i j}\left(-\frac{\beta^{2}}{12 m} \frac{\partial^{2} V}{\partial x_{k}^{2}}+\frac{\beta^{3}}{24 m}\left(\frac{\partial V}{\partial x_{k}}\right)^{2}\right)\right.\right. \\
\left.\left.\quad+\frac{\beta^{2}}{12 m} \frac{\partial^{2} V}{\partial x_{i} \partial x_{j}}\right\}+O\left(\hbar^{4}\right)\right] .
\end{gathered}
$$

Substituting for $C e^{-\beta V}$ to $O\left(\hbar^{2}\right)$ from (26), we find that

$$
P_{i j}=-\left\langle\frac{p_{i}^{\prime} p_{j}^{\prime}}{m}\right\rangle=-n T \delta_{i j}-\frac{\hbar^{2} n}{12 m T} \frac{\partial^{2} V}{\partial x_{i} \partial x_{j}}+O\left(\hbar^{4}\right) .
$$

By setting $i=j$ in (32) and summing, we obtain

$$
W=\frac{1}{2} m n u^{2}+\left\langle\frac{p^{\prime 2}}{2 m}\right\rangle=\frac{3}{2} n T+\frac{1}{2} m n u^{2}+\frac{\hbar^{2} n}{24 m T} \nabla^{2} V+O\left(\hbar^{4}\right) .
$$


At this point, the stress tensor and energy density involve quantum corrections proportional to the second derivatives of the potential energy, and the QHD equations can be formulated in this way. However, Ancona and Tiersten [2] argued on general thermodynamical grounds that the equation of state for a quantum gas near thermal equilibrium should depend on the density gradient (rather than $\nabla V$ ) as well as the density of the gas. In addition, to apply to neutral quantum fluids, e.g., superfluids, the QHD equations should be available in a form valid when the electrostatic potential energy in Poisson's equation is set equal to zero.

In this spirit, we can approximate

$$
\frac{\partial}{\partial x_{i}} \log (n) \approx-\beta \frac{\partial V}{\partial x_{i}}+O\left(\hbar^{2}\right)
$$

from (26) if

$$
\left|\frac{1}{\mathscr{A}} \frac{\partial \mathscr{A}}{\partial x_{i}}\right|, \frac{1}{\beta}\left|\frac{\partial \beta}{\partial x_{i}}\right|,\left|V \frac{\partial \beta}{\partial x_{i}}\right| \ll \beta\left|\frac{\partial V}{\partial x_{i}}\right| .
$$

Equation (34) is, to leading order in $\hbar^{2}$, equivalent to the classical thermal equilibrium expression $n \propto \exp (-\beta V)$ and should be approximately valid for slowly varying $n, \mathbf{u}$, and $T$. Assuming that $\mathscr{A}$ and $T$ are slowly varying according to (35), we can write the stress tensor as

$$
P_{i j}=-n T \delta_{i j}+\frac{\hbar^{2} n}{12 m} \frac{\partial^{2}}{\partial x_{i} \partial x_{j}} \log (n)+O\left(\hbar^{4}\right)
$$

and the energy density as

$$
W=\frac{3}{2} n T+\frac{1}{2} m n u^{2}-\frac{\hbar^{2} n}{24 m} \nabla^{2} \log (n)+O\left(\hbar^{4}\right) .
$$

We use (36) and (37) in this investigation.

Note that both forms for the stress tensor and energy density display the "nonlocal" character of solutions to Schrödinger's equation. In (32) and (33), $P_{i j}$ and $W$ depend on the potential energy not only at $\mathbf{x}$, but in a neighborhood of $\mathbf{x}$, while, in (36) and (37), $P_{i j}$ and $W$ depend on the electron density in a neighborhood of $\mathbf{x}$, as in the quantum potential (6) of Bohm.

The classical equations of compressible fluid dynamics are derived for dilute gases from a moment expansion of the Boltzmann equation by assuming that the distribution function of the gas particles is locally a velocity-shifted Maxwellian and that $n, \mathbf{u}$, and $T$ vary slowly with $\mathbf{x}$ and $t$. However, the resulting conservation laws of compressible fluid dynamics are much more generally valid. Similarly, we expect the QHD equations to be more generally valid than the conditions under which they were derived and to apply to electron flow that is not near thermal equilibrium. In addition to the assumption that the electron flow is near thermal equilibrium, the QHD transport equations were derived assuming that $V$ is sufficiently smooth to have a Taylor series expansion. The potential energy of the resonant tunneling diode, however, has abrupt "jumps" at the $\mathrm{GaAs} / \mathrm{Al}_{x} \mathrm{Ga}_{1-x} \mathrm{As}$ heterojunctions (see Fig. 7). We believe that the QHD equations are valid for this type of potential, as comparison of the current-voltage results of $\$ 6$ with experiment demonstrates. One reason for the extended validity of the QHD equations may be due to the fact that the $\log (n)$ forms (36) and (37) for $P_{i j}$ and $W$ are better able to handle jumps in the potential energy 
than the $V$ forms (32) and (33). The $V$ forms for $P_{i j}$ and $W$ introduce third derivatives of the potential energy into the hydrodynamic transport equations - thus the computed solutions in this case will be extremely sensitive to the exact shape of the computational heterojunction.

The potential energy $V$ for problems like the resonant tunneling diode may be decomposed into a smooth part and a discontinuous part with a finite set of jumps. Odeh and Steinrueck [26] developed an asymptotic, thermal equilibrium solution to the Wigner-Boltzmann equation, treating the discontinuous part of the potential energy separately to obtain a boundary layer solution near the jumps. Future work will apply this approach to deriving quantum fluid dynamical equations that treat the discontinuities in $V$ more rigorously.

As noted above, the thermal equilibrium Wigner distribution function is actually expanded in powers of $\hbar^{2} / 8 m T l^{2}$. Thus the QHD equations approximate the full Wigner-Boltzmann equation when the electron gas is not too far from equilibrium (so that the moment expansion of the Wigner-Boltzmann equation is valid) and in the high temperature limit (in which the expansion of the Wigner distribution function is valid).

For the resonant tunneling diode simulations in $\S 6$ with $T \approx T_{0}=77 \mathrm{~K}$ and $l=100 \AA$, the expansion parameter $\approx 0.23$.

4. Classification of the QHD equations. To classify the QHD equations, we first rewrite (1)-(3) and (8) (with $\tau_{p}, \tau_{w} \rightarrow \infty$ ) as

$$
\begin{gathered}
\frac{\partial n}{\partial t}+\frac{\partial}{\partial x_{i}}\left(n u_{i}\right)=0 \\
\frac{\partial u_{j}}{\partial t}+u_{i} \frac{\partial u_{j}}{\partial x_{i}}+\frac{1}{m n} \frac{\partial}{\partial x_{j}}(n T) \\
-\frac{\hbar^{2}}{12 m^{2} n} \frac{\partial}{\partial x_{i}}\left(n \frac{\partial^{2}}{\partial x_{i} \partial x_{j}} \log (n)\right)+\frac{1}{m} \frac{\partial V}{\partial x_{j}}=0 \\
\frac{\partial T}{\partial t}+u_{i} \frac{\partial T}{\partial x_{i}}+\frac{2}{3} T \frac{\partial u_{i}}{\partial x_{i}}-\frac{2}{3 n} \frac{\partial}{\partial x_{i}}\left(\kappa \frac{\partial T}{\partial x_{i}}\right)+\frac{\hbar^{2}}{36 m n} \frac{\partial}{\partial x_{i}}\left(n \nabla^{2} u_{i}\right)=0 \\
-\nabla \cdot(\epsilon \nabla V)+e^{2}\left(N_{D}-N_{A}-n\right)=0
\end{gathered}
$$

Next, we perturb a solution of the QHD equations with a Fourier mode, linearize the partial differential equations (PDEs) (38)-(41) with respect to the perturbation, and freeze coefficients. Set

$$
\left[\begin{array}{c}
n \\
u_{i} \\
T \\
V
\end{array}\right]=\left[\begin{array}{c}
\bar{n} \\
\overline{u_{i}} \\
\bar{T} \\
\bar{V}
\end{array}\right]+e^{-\sigma t+i \mathbf{k} \cdot \mathbf{x}}\left[\begin{array}{c}
\delta n \\
\delta u_{i} \\
\delta T \\
\delta V
\end{array}\right],
$$

where $\left[\bar{n}, \overline{u_{i}}, \bar{T}, \bar{V}\right]$ is a solution of the QHD equations. We write the linearized QHD equations as

$$
-\operatorname{diag}\{\sigma, \sigma, \sigma, \sigma, \sigma, 0\}\left[\delta n, \delta u_{i}, \delta T, \delta V\right]+\mathscr{S}\left[\delta n, \delta u_{i}, \delta T, \delta V\right]=0
$$


where $\mathscr{S}$ is the symbol of the linearized PDE system (38)-(41). Asymptotically as $|\mathbf{k}| \rightarrow \infty, \mathscr{S}-\operatorname{diag}\left\{\sigma, \sigma \delta_{i j}, \sigma, 0\right\}$ has the form

$$
j \downarrow\left[\begin{array}{cccc}
i \mathbf{k} \cdot \mathbf{u}-\sigma & i k_{i} n & 0 & 0 \\
i k_{j} \frac{T}{m n}+i k_{j} k^{2} \frac{\hbar^{2}}{12 m^{2} n} & (i \mathbf{k} \cdot \mathbf{u}-\sigma) \delta_{i j} & i \frac{k_{j}}{m} & i \frac{k_{j}}{m} \\
i k_{i} \frac{\hbar^{2}}{36 m n} \nabla^{2} u_{i} & \frac{2}{3} i k_{i} T-i k_{i} k^{2} \frac{\hbar^{2}}{36 m} & i \mathbf{k} \cdot \mathbf{u}+\frac{2}{3} k^{2} \frac{\kappa}{n}-\sigma & 0 \\
0 & 0 & 0 & k^{2} \epsilon
\end{array}\right],
$$

where $i$ labels columns in the velocity perturbation $\delta u_{i}$ and $j$ labels rows in the velocity equation (39). The bar over the solution has been dropped for clarity of notation, and the leading terms in $\mathbf{k}$ for the limits $\hbar \rightarrow 0$ and $\kappa \rightarrow 0$ have been separately kept.

The asymptotic eigenvalues $\sigma$ of the symbol as $|\mathbf{k}| \rightarrow \infty$ determine the mathematical type of the PDE system. The eigenvalues to leading order in $\mathbf{k}$ can most easily be deduced from the trace and determinant of the symbol. In fact, we need only consider the upper $5 \times 5$ block $\mathscr{S}_{5}$ of the symbol, since the coupling of the transport equations (38)-(40) to Poisson's equation (41) only introduces the elliptic Poisson mode and does not affect the modes of the transport equations.

There are three physically interesting cases to consider.

Case 1. $\hbar=0, \quad \kappa=0$ (electrogasdynamics). $\operatorname{Tr} \mathscr{S}_{5}=5 i \mathbf{k} \cdot \mathbf{u}$ and $\operatorname{det} \mathscr{S}_{5}=$ $-i(\mathbf{k} \cdot \mathbf{u})^{3}\left(k^{2} c^{2}-(\mathbf{k} \cdot \mathbf{u})^{2}\right)$, where $c=\sqrt{5 T / 3 m}$. The eigenvalues of the symbol $\mathscr{S}_{5}$ and corresponding modes are

$$
\begin{array}{ll}
i(\mathbf{k} \cdot \mathbf{u} \pm k c) & \text { hyperbolic, } \\
i \mathbf{k} \cdot \mathbf{u} & \text { hyperbolic, multiplicity } 3 .
\end{array}
$$

There are five nonlinear waves in classical electrogasdynamics corresponding to the five hyperbolic modes: two shock waves with characteristic speeds $u_{n} \pm c$, where $u_{n}$ is the velocity normal to the wave, and three contact waves with characteristic speed $u_{n}$. Two contact waves can be labeled by a jump in the tangential velocity $\mathbf{u}_{t}$ across the wave, and one contact wave by a jump in the temperature $T$.

Case 2. $\hbar=0, \kappa>0$ (hydrodynamic model or electrogasdynamics with heat conduction). $\operatorname{Tr} \mathscr{S}_{5}=\frac{2}{3} k^{2} \kappa / n+5 i \mathbf{k} \cdot \mathbf{u}$ and $\operatorname{det} \mathscr{S}_{5}=-\frac{2}{3} k^{2}(\kappa / n)(\mathbf{k} \cdot \mathbf{u})^{2}\left(k^{2} c^{2}-\right.$ $\left.(\mathbf{k} \cdot \mathbf{u})^{2}\right)-i(\mathbf{k} \cdot \mathbf{u})^{2}\left(k^{2} c^{2}-(\mathbf{k} \cdot \mathbf{u})^{2}\right)$, where $c=\sqrt{T / m}$. The eigenvalues of the symbol $\mathscr{S}_{5}$ and corresponding modes are

$$
\begin{array}{ll}
i(\mathbf{k} \cdot \mathbf{u} \pm k c) & \text { hyperbolic, } \\
i \mathbf{k} \cdot \mathbf{u} & \text { hyperbolic, multiplicity } 2, \\
\frac{2}{3} k^{2} \kappa / n+i \mathbf{k} \cdot \mathbf{u} & \text { parabolic. }
\end{array}
$$

With heat conduction, there are four nonlinear waves [13] in the classical hydrodynamic model corresponding to the four hyperbolic modes: two shock waves with characteristic speeds $u_{n} \pm c$ and two contact waves with characteristic speed $u_{n}$. The two contact waves can be labeled by a jump in the tangential velocity $\mathbf{u}_{t}$ across the 
wave. The contact wave corresponding to a discontinuity in $T$ has disappeared due to the parabolic heat conduction term $\nabla \cdot(\kappa \nabla T)$ in (3).

Case 3. $\hbar \neq 0, \quad \kappa>0$ (QHD model). $\operatorname{Tr} \mathscr{S}_{5} \approx \frac{2}{3} k^{2} \kappa / n$ and $\operatorname{det} \mathscr{S}_{5} \approx$ $-(\mathbf{k} \cdot \mathbf{u})^{2} k^{6} \hbar^{2} \kappa / 18 m^{2} n$. The eigenvalues of the symbol $\mathscr{S}_{5}$ and corresponding modes are

$$
\begin{array}{ll} 
\pm i k^{2} \frac{\hbar}{\sqrt{12} m} & \text { Schrödinger, } \\
i \mathbf{k} \cdot \mathbf{u} & \text { hyperbolic, multiplicity 2, } \\
\frac{2}{3} k^{2} \kappa / n & \text { parabolic. }
\end{array}
$$

There are two contact discontinuities (in $\mathbf{u}_{t}$ ) in the QHD model corresponding to the two hyperbolic modes. Note that two of the hyperbolic modes that allow shock discontinuities to form in the classical hydrodynamic model have become Schrödinger modes when the quantum corrections are included.

Well-posed boundary conditions for the two-dimensional (2D) (and by extension 3D) classical hydrodynamic model are formulated in [20], assuming subsonic flow at the inflow and outflow boundaries. In one dimension, the QHD model with heat conduction has two Schrödinger modes, one parabolic mode, and one elliptic mode. Thus eight boundary conditions are necessary. Well-posed boundary conditions for the resonant tunneling diode are $n=N_{D}, \partial n / \partial x=0$, and $T=T_{0}$ at $x_{\min }$ and $x_{\max }$, with a bias $\Delta V$ across the device: $V\left(x_{\text {min }}\right)=T \log \left(n / n_{i}\right)$ and $V\left(x_{\text {max }}\right)=T \log \left(n / n_{i}\right)$ $+e \Delta V$, where $n_{i}$ is the intrinsic electron concentration.

5. The second upwind method for the 1D QHD model. In this section, the 1D steady-state QHD equations are discretized using an upwind method adapted from computational fluid dynamics. ${ }^{4}$ Since the upwind method requires velocity values $u_{-1 / 2}, u_{1 / 2}, u_{3 / 2}, \ldots, u_{N-1 / 2}, u_{N+1 / 2}$ at the midpoints of the elements $l_{i}(i=1, \ldots, N)$ connecting grid points $i-1$ and $i$, a staggered grid is used for $u$. (A computational Neumann boundary condition $d u / d x=0$ at $i=0$ and $i=N$ is imposed to determine $u_{-1 / 2}$ and $u_{N+1 / 2}$.) Defining the velocities at midpoints of elements also resolves waves more sharply by avoiding the averaging of velocities defined at grid points $i$, $i \pm 1$ to obtain $u_{i-1 / 2}$ and $u_{i+1 / 2}$. The variables $n, T$, and $V$ are defined at the grid points $i=0,1, \ldots, N-1, N$. The boundary conditions specify $n, d n / d x, T$, and $V$ at $i=0$ and $i=N$. (The boundary conditions for $d n / d x$ at $i=0$ and $i=N$ specify values for $n_{-1}$ and $n_{N+1}$ that appear in the upwind discretization.)

In one dimension, the steady-state QHD model consists of the three nonlinear conservation laws for electron number, momentum, and energy, plus Poisson's equation

$$
\left[\begin{array}{c}
f_{n} \\
f_{u} \\
f_{T} \\
f_{V}
\end{array}\right]=\frac{d}{d x}\left[\begin{array}{c}
u g_{n} \\
u g_{u} \\
u g_{T} \\
0
\end{array}\right]+\left[\begin{array}{c}
0 \\
h_{u} \\
h_{T} \\
h_{V}
\end{array}\right]+\left[\begin{array}{c}
0 \\
s_{u} \\
s_{T} \\
s_{V}
\end{array}\right]=0
$$

\footnotetext{
${ }^{4}$ The second upwind method was applied to hydrodynamic model simulations of electron shock waves in submicron $n^{+}-n-n^{+}$devices in [10]-[12].
} 
where

$$
\begin{gathered}
g_{n}=n, \\
g_{u}=m n u \\
g_{T}=\frac{5}{2} n T+\frac{1}{2} m n u^{2}-\frac{\hbar^{2} n}{8 m} \frac{d^{2}}{d x^{2}} \log (n)+n V \\
h_{u}=\frac{d}{d x}(n T)-\frac{d}{d x}\left(\frac{\hbar^{2} n}{12 m} \frac{d^{2}}{d x^{2}} \log (n)\right)+n \frac{d V}{d x} \\
h_{T}=-\frac{d}{d x}\left(\kappa \frac{d T}{d x}\right) \\
h_{V}=\epsilon \frac{d^{2} V}{d x^{2}} \\
s_{u}=\frac{m n u}{\tau_{p}} \\
s_{T}=\left(\frac{3}{2} n T+\frac{\hbar^{2} n}{2} m n u^{2}-\frac{d^{2}}{24 m} \log (n)-\frac{3}{2} n T_{0}\right) / \tau_{w}, \\
s_{V}=e^{2}\left(N_{D}-N_{A}-n\right) .
\end{gathered}
$$

Equations $f_{n}=0, f_{T}=0$, and $f_{V}=0$ are enforced at the interior grid points $i=1, \ldots, N-1$, while equation $f_{u}=0$ is enforced at the midpoints of the elements $l_{i}$, $i=1, \ldots, N$.

In the second upwind method, ${ }^{5}$ the advection terms $d(u g) / d x$ in (48) are discretized using second upwind differences

$$
\frac{d}{d x}(u g)_{i} \approx\left(u_{i+1 / 2} g_{R}-u_{i-1 / 2} g_{L}\right) / \Delta x
$$

where

$$
g_{R}=\left\{\begin{array}{ll}
g_{i} & \left(u_{i+1 / 2}>0\right), \\
g_{i+1} & \left(u_{i+1 / 2}<0\right),
\end{array} \quad g_{L}= \begin{cases}g_{i-1} & \left(u_{i-1 / 2}>0\right), \\
g_{i} & \left(u_{i-1 / 2}<0\right),\end{cases}\right.
$$

and second-order central differences are used for $h_{u}, h_{T}, h_{V}$, and $s_{T}$.

To linearize the discretized version of (48), we use Newton's method

$$
J\left[\begin{array}{l}
\delta n \\
\delta u \\
\delta T \\
\delta V
\end{array}\right]=-\left[\begin{array}{l}
f_{n} \\
f_{u} \\
f_{T} \\
f_{V}
\end{array}\right]=-f, \quad\left[\begin{array}{l}
n \\
u \\
T \\
V
\end{array}\right] \leftarrow\left[\begin{array}{l}
n \\
u \\
T \\
V
\end{array}\right]+t\left[\begin{array}{l}
\delta n \\
\delta u \\
\delta T \\
\delta V
\end{array}\right],
$$

where $J$ is the Jacobian aind $t$ is a damping factor [4] between 0 and 1 , chosen to insure that the norm of the residual $f$ decreases monotonically.

${ }^{5}$ The second upwind method is a conservative extension of the original first-order upwind method; see, e.g., [24]. 
6. QHD simulations of the resonant tunneling diode. The QHD model allows efficient simulation of the behavior of quantum devices that depend on particle tunneling through potential barriers and charge buildup in potential wells. Simulations of a GaAs resonant tunneling diode with double $\mathrm{Al}_{x} \mathrm{Ga}_{1-x}$ As barriers are presented here. The barrier height $\mathscr{B}$ is set equal to $0.209 \mathrm{eV}$ (the $\mathrm{Al}$ mole fraction $x=0.3$ ). The diode consists of $n^{+}$source (at the left) and drain (at the right) regions with the doping density $N_{D}=10^{18} \mathrm{~cm}^{-3}$ and an $n$ channel with $N_{D}=5 \times 10^{15} \mathrm{~cm}^{-3}$ (see Fig. 1). A hyperbolic tangent fitting is used for $N_{D}$ over $\pm 50 \AA$ at the junctions. The channel is $250 \AA$ long as indicated by the vertical lines at $x=0$ and $x=0.025$ microns in Figs. $3,4,6$, and 7; the barriers are $50 \AA$ wide as indicated by the light gray bands in Figs. 1, 3, 4, 6, and 7; and the quantum well between the barriers is $50 \AA$ wide. Note that the device has $50 \AA$ spacers between the barriers and the contacts (source and drain).

The barrier height $\mathscr{B}$ is incorporated into the QHD transport equations (1)-(3) by replacing $V \rightarrow V+\mathscr{B}$. (Poisson's equation is not changed.)

We use modified Baccarani-Wordemann models [3], [12] for the classical momentum and energy relaxation times in the QHD model

$$
\begin{gathered}
\tau_{p}=\tau_{p 0} \frac{T_{0}}{T}, \\
\tau_{w}=\frac{\tau_{p}}{2}\left(1+\frac{\frac{3}{2} T}{\frac{1}{2} m v_{s}^{2}}\right),
\end{gathered}
$$

where the low-energy momentum relaxation time $\tau_{p 0}$ is set equal to $0.9 \mathrm{ps}$ from the low-field electron mobility in GaAs at $77 \mathrm{~K}$. The low-field electron mobility $\mu_{n 0}$ is related to $\tau_{p 0}$ by $\tau_{p 0}=m \mu_{n 0} / e$, where $\mu_{n 0}=, 25,000 \mathrm{~cm}^{2} / \mathrm{Vs}$ (see [28] and [7]). The effective electron mass $m=0.063 m_{e}$, where $m_{e}$ is the electron mass, and the saturation velocity $v_{s} \approx 2 \times 10^{7} \mathrm{~cm} / \mathrm{s}$ for lower valley electrons in GaAs at $77 \mathrm{~K}$. The dielectric constant $\epsilon=12.9$ for GaAs.

Continuation in voltage $\Delta V$ was found to be necessary in generating the solutions: that is, to find the solution at $\Delta V=0.1$ volts, for example, the solutions are

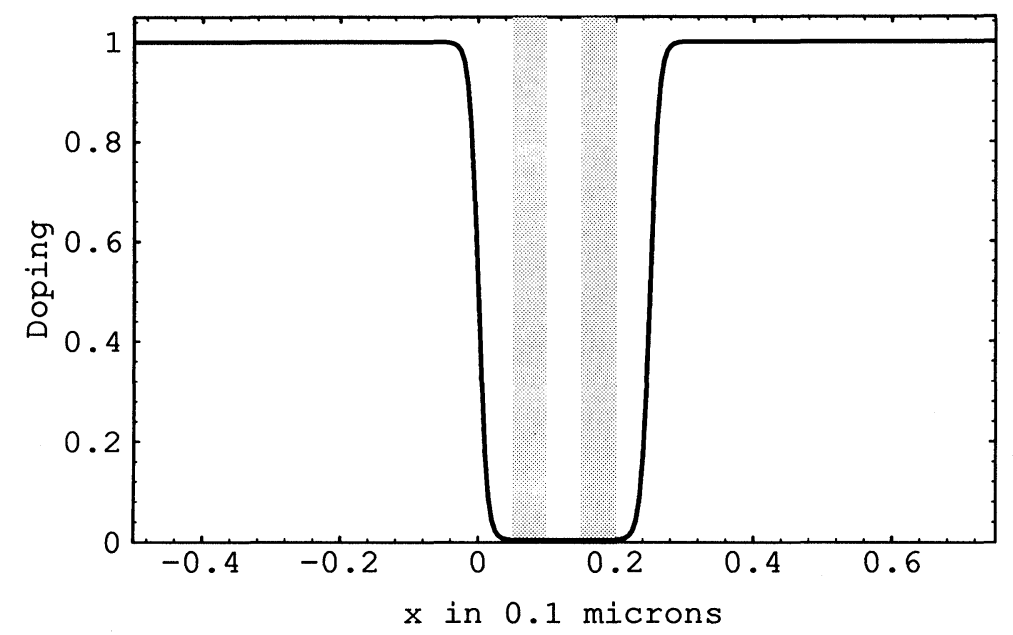

FIG. 1. Doping $/ 10^{18} \mathrm{~cm}^{-3}$. 
generated at $\Delta V_{1}=0.001$ volts, $\Delta V_{2}=0.002$ volts, .., at each stage using the previous solution at $\Delta V_{j}$ as the initial guess for the Newton method in computing the solution at $\Delta V_{j+1}$. An algorithm for choosing the $\Delta V_{j}^{\prime}$ 's is used that attempts to maximize the voltage increments while keeping the number of Newton iterations small. Typical simulations on a grid with $250 \Delta x$ require 1-3 minutes of CPU time on a Sun SPARCstation 10.

Current-voltage curves for the resonant tunneling diode at $77 \mathrm{~K}$ are plotted in Fig. 2 for three different values of $\kappa_{0}$ in the Wiedemann-Franz expression for thermal conductivity

$$
\mathbf{q}=-\kappa \nabla T, \quad \kappa=\kappa_{0} \tau_{p 0} n T_{0} / m
$$

The region of negative differential resistance extends from the peak to the valley of the current-voltage curve. These are the first simulations of the full QHD equations to show NDR in the resonant tunneling diode.

The peak of the current-voltage curve occurs as the electrons tunneling through the first barrier come into resonance with the ground state of the quantum well. Note the presence of a "shoulder" in the current-voltage curve around $\Delta V=0.25$ volts (cf. [29]). The shoulder signals the location of the first virtual state of the quantum well. The location of the peak and shoulder can be qualitatively understood from the energy levels of a square well. For an infinite well, $E_{n}=(n+1)^{2} \pi^{2} \hbar^{2} / 2 m x_{0}^{2}$, where $x_{0}$ is the width of the well and where $n$ is a nonnegative integer. For a $50 \AA$ wide GaAs infinite well, the ground state energy $E_{0}=0.24 \mathrm{eV}$ and the energy of the first excited state $E_{1}=0.96 \mathrm{eV}$. For a $50 \AA$ wide $0.209 \mathrm{eV}$ high $\mathrm{GaAs}$ finite square well (see, e.g., Leighton [18]), there is just one bound state, and the energy level is shifted to $E_{0}=0.079 \mathrm{eV}$. The energy of the first virtual state of the well is $0.24 \mathrm{eV}$.

Note that the main effect of larger values of $\kappa_{0}$ is to shift the peak of the current-voltage curve to the right. With lower values of $\kappa_{0}$, the electrons have a

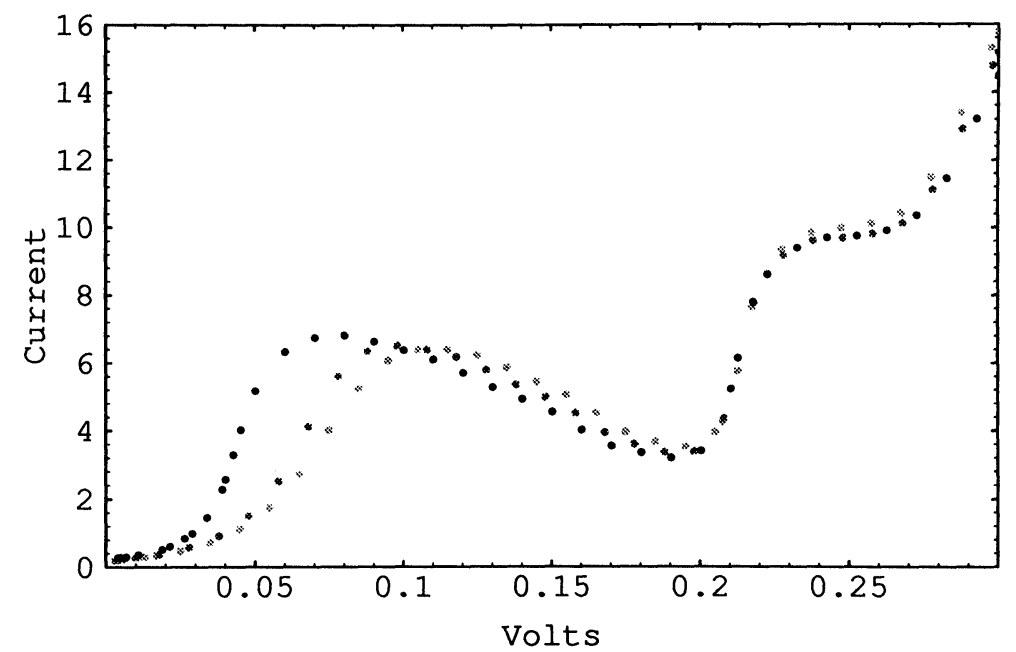

FIG. 2. Current density in kiloamps $/ \mathrm{cm}^{2}$ versus voltage for the resonant tunneling diode at $77 \mathrm{~K} . \kappa_{0}=0.2$ (black), 0.4 (gray), and 0.6 (light gray). The dots represent computed solution points. 
higher average energy as they impinge upon the first barrier, and therefore resonate with the well at a lower applied voltage.

A physically relevant value of $\kappa_{0}$ is approximately 0.4 for this device. The peak-to-valley current ratio of 1.95 agrees quantitatively with experimental ratios for similar devices (see, e.g., [21], [23]). In fact, for the device parameters of [23], the QHD code predicts a peak-to-valley current ratio of 2.65 (with $\kappa_{0}=1$ ), compared with the experimental value of approximately 2.5. The experimental (computational) peak occurs at $\Delta V=0.18(0.11)$ volts, and the experimental (computational) valley occurs at $\Delta V=0.35(0.30)$ volts.

Figures 3-7 illustrate the electron density, velocity, and temperature, and the conduction band energy in the resonant tunneling diode for $\kappa_{0}=0.4$. Figure 3 shows the dramatic charge enhancement in the quantum well typical of the resonant tunneling diode for applied voltages of $\Delta V=0.097$ (peak), 0.191 (valley), and 0.22 volts (just before the shoulder). The electron density at the center of the quantum well increases as $\Delta V$ increases and is more than two orders of magnitude larger than the background doping density. Note the depletion of electrons around the channeldrain junction. Figure 4 presents the electron velocities for the same set of applied voltages. Of special note is the fact that the lowest velocity in the well occurs at the voltage $\Delta V_{\text {valley }}$ at which the current-voltage valley occurs. The electrons spend significantly more time in the quantum well (where the velocity is low) than in the barriers (where the velocity is high) [14].

As illustrated in Fig. 5, the electrons spend the longest time in the quantum well for voltages near $\Delta V_{\text {valley }}$. The "dwell" time spent by electrons in the well increases monotonically up to voltages near $\Delta V_{\text {valley }}$ and then decreases rapidly. Note that the macroscopic QHD dwell time differs qualitatively from dwell times based on microscopic quantum calculations, which predict that the dwell time is maximum at resonance [5], [6], [16].

The electrons cool down to 0.14 ( $\Delta V=0.097$ volts $)$ to 0.35 ( $\Delta V=0.22$ volts $)$ times $T_{0}$ as they overcome the first $\mathrm{Al}_{x} \mathrm{Ga}_{1-x}$ As potential barrier (see Fig. 6). As the electrons are accelerated near the channel-drain junction, they heat up to $2(\Delta V=$ 0.097 volts) to 3.5 ( $\Delta V=0.22$ volts) times $T_{0}$ and then cool back down to the lattice

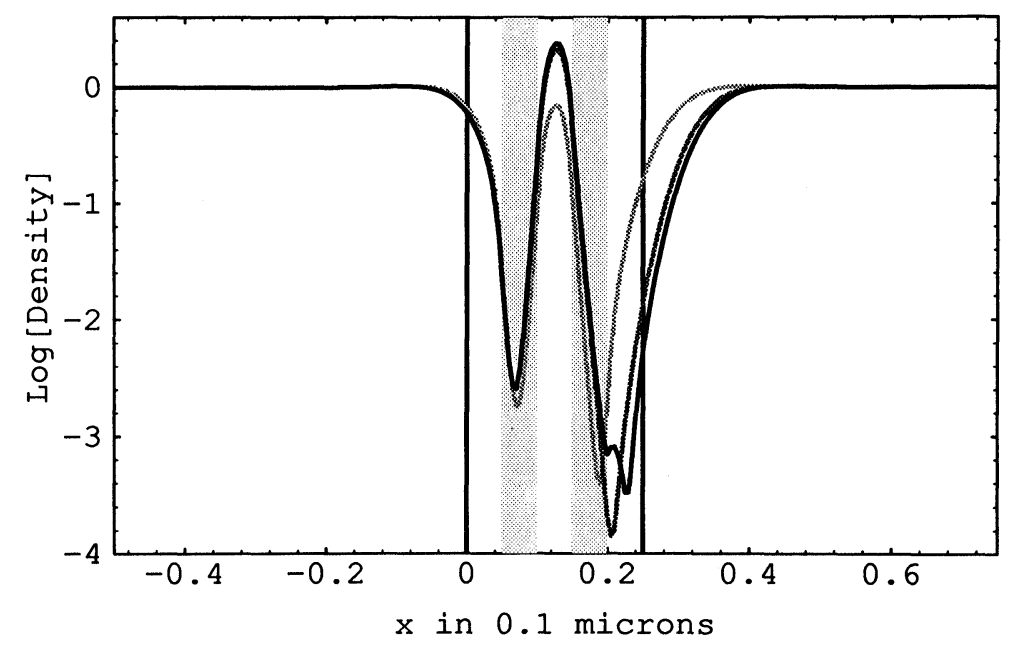

Fig. 3. $\log$ (Density $/ 10^{18} \mathrm{~cm}^{-3}$ ). The curves are for $\Delta V=0.097$ (light gray), 0.191 (gray), and 0.22 (black) volts. 


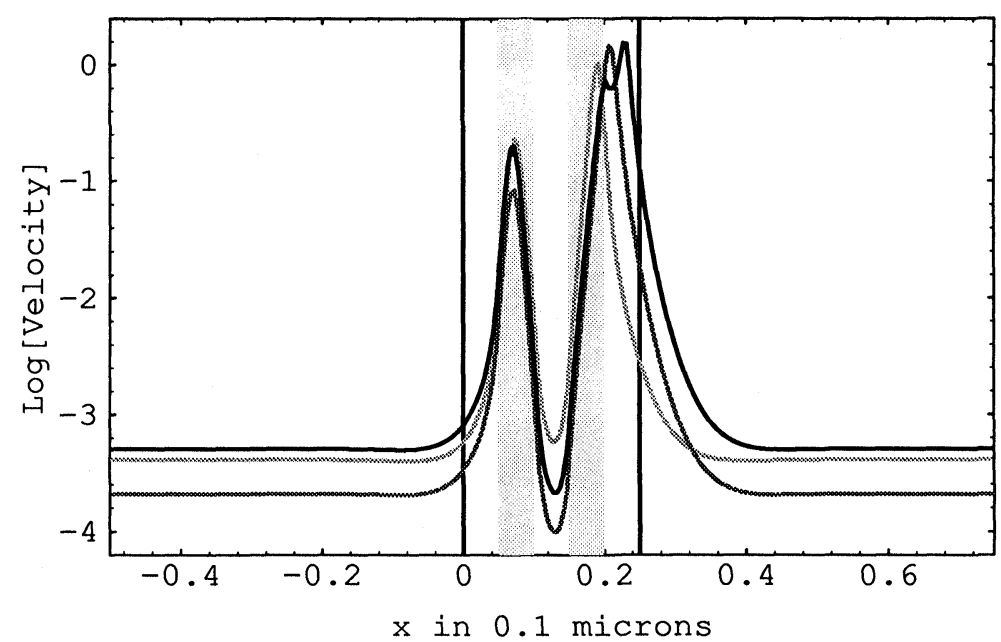

Fig. 4. $\log$ (Velocity $/ 10^{8} \mathrm{~cm} / \mathrm{sec}$ ). The curves are for $\Delta V=0.097$ (light gray), 0.191 (gray), and 0.22 (black) volts.

temperature in the drain. The conduction band energies $V+\mathscr{B}$ are illustrated in Fig. 7 and show substantial band bending in the well and barriers.

7. Conclusion. The QHD model offers several advantages over other methods for simulating quantum semiconductor devices. Although the Wigner function [17] or density matrix [8] methods for simulating the resonant tunneling diode include quantum effects to all orders in $\hbar$, the QHD method is much less computationally intensive and includes the same physics if the expansion parameter $\hbar^{2} / 8 m T l^{2}$ is small. The more complete quantum mechanical methods also have technical drawbacks: The density matrix has "off-diagonal" terms that are difficult to interpret physically and requires the computation of a set of eigenvalues and eigenfunctions,

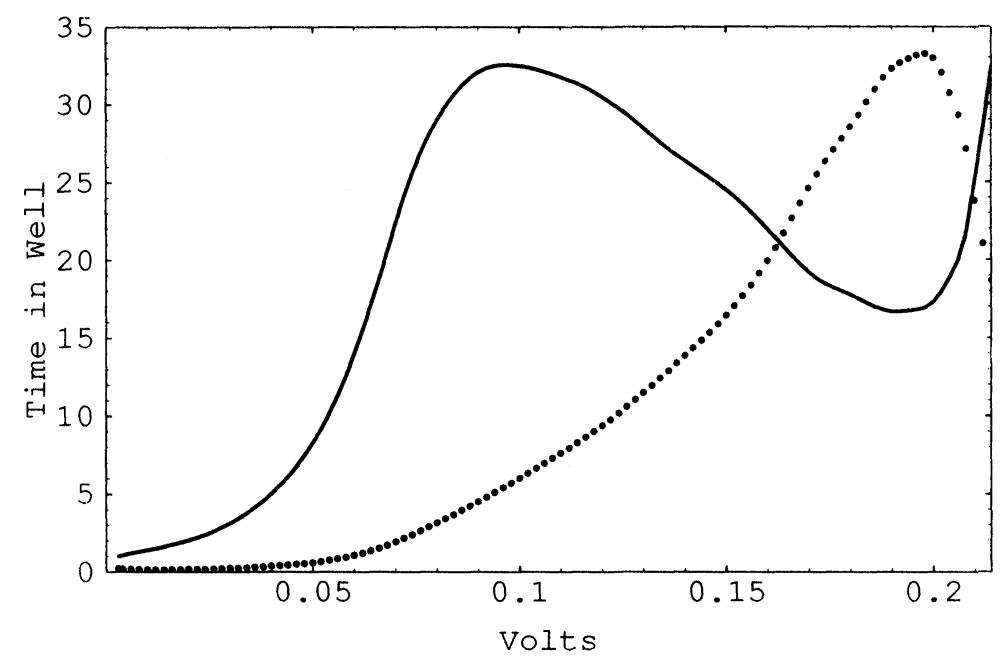

FIG. 5. Time in picoseconds (dots) spent by electrons in the quantum well versus voltage. For reference, a scaled version of the current density (solid line) is also shown. 


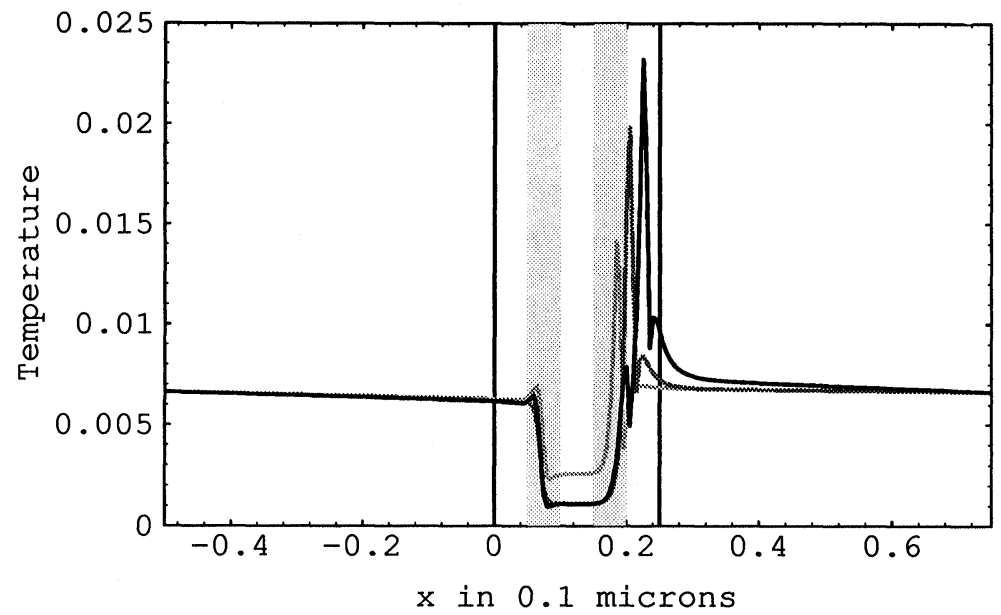

Fig. 6. Temperature in $\mathrm{eV}$. The lattice temperature $T_{0}=77 \mathrm{~K}=0.0066 \mathrm{eV}$. The curves are for $\Delta \mathrm{V}=0.097$ (light gray), 0.191 (gray), and 0.22 (black) volts.

while the Wigner function method requires complicated initial conditions for simulations.

Since the QHD equations have the same form as the classical fluid dynamical equations, well-understood classical boundary conditions can be applied in simulating quantum devices. How to impose proper boundary conditions for full quantum mechanical approaches is still an open question (see [9]). Moreover, the QHD equations are expressed in terms of the fluid dynamical quantities density, velocity, and temperature. These classical fluid dynamical concepts enable us to interpret electron behavior in quantum devices in a physically intuitive way. We can define the time spent by an electron in the quantum well or the electron temperature throughout the device in a precise manner. In turn, the intuitive understanding developed through the QHD model sheds light on more fully quantum mechanical descriptions of electron behavior in quantum devices.

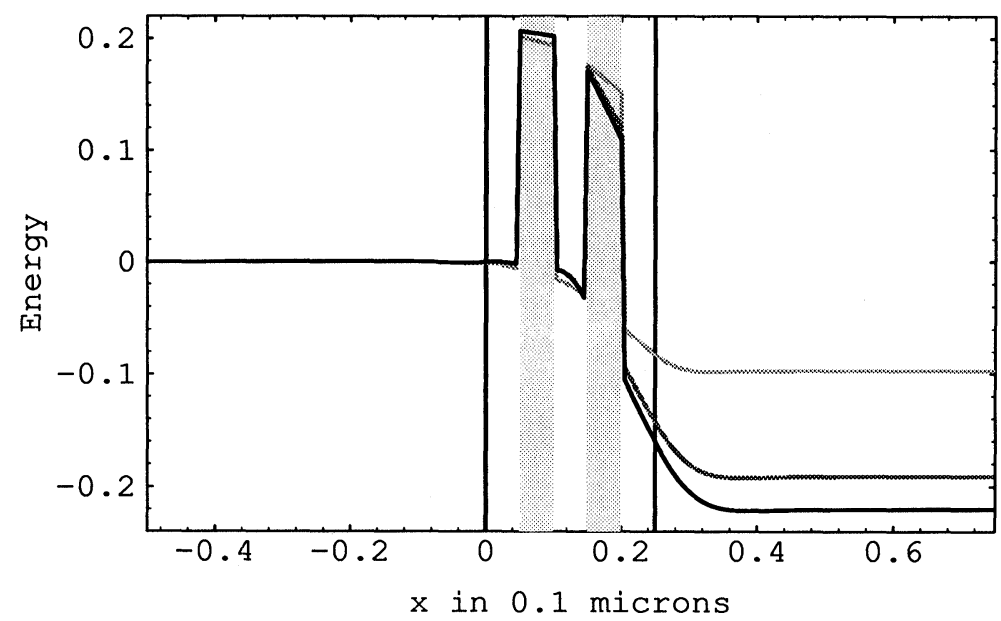

FIG. 7. Conduction band energy in $\mathrm{eV}$. The curves are for $\Delta V=0.097$ (light gray), 0.191 (gray), and 0.22 (black) volts. 
Acknowledgments. This article is dedicated to the memory of my friend and colleague Farouk Odeh, who taught me many things about the classical hydrodynamic model. Farouk was very much interested in the QHD model, and I benefitted from our conversations on this topic.

I would also like to thank Patrick Hagan, David Schaeffer, Stephen Teitsworth, Peter Turley, and Dwight Woolard for valuable discussions.

\section{REFERENCES}

[1] M. G. ANCONA AND G. J. IAfRATE, Quantum correction to the equation of state of an electron gas in a semiconductor, Phys. Rev. B, 39 (1989), pp. 9536-9540.

[2] M. G. Ancona And H. F. Tiersten, Macroscopic physics of the silicon inversion layer, Phys. Rev. B, 35 (1987), pp. 7959-7965.

[3] G. BACCARANI AND M. R. WORDEMAN, An investigation of steady-state velocity overshoot effects in $\mathrm{Si}$ and GaAs devices, Solid State Electronics, 28 (1985), pp. 407-416.

[4] R. E. Bank AND D. J. Rose, Global approximate Newton methods, Numer. Math., 37 (1981), pp. 279-295.

[5] J. R. BARKER, Quantum theory of hot electron tunneling in microstructures, Physica B, 134 (1985), pp. 22-31.

[6] S. Collins, D. Lowe, AND J. R. BARKer, The quantum mechanical time problem-revisited, J. Phys. C, 20 (1987), pp. 6213-6232.

[7] M. V. FischETTI, Monte Carlo simulation of transport in technologically significant semiconductors of the diamond and zinc-blende structures-Part I: Homogeneous transport, IEEE Trans. Electron Devices, 38 (1991), pp. 634-649.

[8] W. R. FrensLey, Simulation of resonant-tunneling heterostructure devices, J. Vacuum Sci. Tech., B3 (1985), pp. 1261-1266.

[9] - Boundary conditions for open quantum systems driven far from equilibrium, Rev. Modern Phys., 62 (1990), pp. 745-791.

[10] C. L. GARDNER, Numerical simulation of a steady-state electron shock wave in a submicrometer semiconductor devices, IEEE Trans. Electron Devices, 38 (1991), pp. 392-398.

[11] — - Hydrodynamic and Monte Carlo simulation of an electron shock wave in a one micrometer $n^{+}-n-n^{+}$diode, IEEE Trans. Electron Devices, 40 (1993), pp. 455-457.

[12] - Shock waves in the hydrodynamic model for semiconductor devices, in Semiconductors, vol. 58, IMA Vol. Math. Appl., Springer-Verlag, New York, 1993.

[13] C. L. GARDNER, J. W. JEROME, AND D. J. Rose, Numerical methods for the hydrodynamic device model: Subsonic flow, IEEE Trans. on Computer-Aided Design of Integrated Circuits and Systems, 8 (1989), pp. 501-507.

[14] H. L. Grubin AND J. P. KResKovsky, Quantum moment balance equations and resonant tunnelling structures, Solid-State Electronics, 32 (1989), pp. 1071-1075.

[15] G. J. IAfrate, H. L. Grubin, AND D. K. FerRY, Utilization of quantum distribution functions for ultra-submicron device transport, J. de Physique, Colloque C7, 42 (1981), pp. 307-312.

[16] A. P. JAUHO AND M. M. NIETO, Time-dependent tunneling of wave-packets through heterostructures in an applied field, Superlattices and Microstructures, 2 (1986), pp. 407-413.

[17] N. C. Kluksdahl, A. M. Kriman, D. K. Ferry, and C. Ringhofer, Self-consistent study of the resonant-tunneling diode, Phys. Rev. B, 39 (1989), pp. 7720-7735.

[18] R. B. Leighton, Principles of Modern Physics, McGraw-Hill, New York, 1959.

[19] O. MAdelung, Introduction to Solid-State Theory, Springer-Verlag, Berlin, 1978.

[20] F. Odeh AND E. Thomann, On the well-posedness of the two-dimensional hydrodynamic model for semiconductor devices, COMPEL, 9 (1990), pp. 45-47.

[21] H. Ohnishi, T. Inata, S. Muto, N. Yokoyama, AND A. Shibatomi, Self-consistent analysis of resonant tunneling current, Appl. Phys. Lett., 49 (1986), pp. 1248-1250.

[22] C. Philippidis, D. Bohm, AND R. D. Kaye, The Aharonov-Bohm effect and the quantum potential, Il Nuovo Cimento, 71B (1982), pp. 75-88.

[23] S. Ray, P. Ruden, V. Sokolov, R. Kolbas, T. Boonstra, and J. Williams, Resonant tunneling transport at $300 \mathrm{~K}$ in GaAs-AlGaAs quantum wells grown by metalorganic chemical vapor deposition, Appl. Phys. Lett., 48 (1986), pp. 1666-1668. 
[24] P. J. RoAche, Computational Fluid Dynamics, Hermosa, Albuquerque, NM, 1982.

[25] M. RUdAN AND F. ODEH, Multi-dimensional discretization scheme for the hydrodynamic model of semiconductor devices, COMPEL, 5 (1986), pp. 149-183.

[26] H. Steinrueck AND F. Odeh, The Wigner function for thermal equilibrium, Z. Angew. Math. Phys., 42 (1991), pp. 470-487.

[27] M. A. STroscio, Moment-equation representation of the dissipative quantum Liouville equation, Superlattices and Microstructures, 2 (1986), pp. 83-87.

[28] M. Tomizawa, K. Yokoyama, AND A. YoshiI, Hot-electron velocity characteristics at AlGaAs $/ \mathrm{GaAs}$ heterostructures, IEEE Electron Device Letters, EDL-5 (1984), pp. 464-465.

[29] P. J. TURLEY AND S. W. TeITSWORTH, Electronic wave functions and electron-confined-phonon matrix elements in $\mathrm{GaAs} / \mathrm{Al}_{x} \mathrm{Ga}_{1-x} \mathrm{As}$ double-barrier resonant tunneling structures, Phys. Rev. B, 44 (1991), pp. 3199-3210.

[30] G. B. Whitham, Linear and Nonlinear Waves, John Wiley, New York, 1974.

[31] E. Wigner, On the quantum correction for thermodynamic equilibrium, Phys. Rev., 40 (1932), pp. 749-759.

[32] D. L. Woolard, M. A. Stroscio, M. A. Littlejohn, R. J. Trew, and H. L. Grubin, A new nonparabolic hydrodynamic model with quantum corrections, in Computational Electronics: Semiconductor Transport and Device Simulation, Kluwer Academic Publishers, Boston, 1991.

[33] D. L. Woolard, H. Tian, M. A. Littlejohn, K. W. Kim, R. J. Trew, M. K. Ieong, and T. W. Tang, The construction of higher-moment terms in the hydrodynamic electron transport model, J. Appl. Phys., to appear.

[34] J.-R. ZHOU AND D. K. FERRY, Simulation of ultra-small GaAs MESFET using quantum moment equations, IEEE Trans. Electron Devices, 39 (1992), pp. 473-478. 